\title{
69
}

\section{TWO FURTHER NOTES ON THE ORIGIN OF DOMINANCE}

SINCE the publication in April, 1928, of my calculations of "The possible modification of the response of the wild type to recurrent mutations," (THE AMERICAN NATURAUST, LXII, pp. 115126) the interest of a number of geneticists, some of whom were personally unknown to me, has brought many further facts into the discussion, in the light of which the somewhat tentative tone of that paper seems to have been unnecessary.

(a) The first point, the facts of which I owe to Mr. J. B. Hutchinson, arose in some investigations into the genetics of the cotton plant, by Dr. S. C. Harland, which I understand are to be published during this year. The case illustrates well how decisive evidence may sometimes come from an entirely unforeseen quarter; for it provides the most direct demonstration possible of the acquirement of dominance in response to a mutation.

It appears that a mutant form, Crinkled Dwarf, which occurs in the Sea Island cottons, is, in that species, a simple recessive, while in other New World species it is not known to occur. These species, moreover, are mutually quite fertile, and therefore the mutant gene can be introduced into any of them by crossing. Dr. Harland has done this with at least two other New World cotton species, with the same result. The $F_{1}$ plants were slightly modified by the mutant, showing even at this stage some incompleteness of dominance. In the $F_{2}$, formed by self-fertilization, every degree of dominance seems to have appeared in a quite unclassifiable series. It can scarcely be doubted therefore that the Sea Island cottons, among which this mutant appears, differ from other New World species in a number of modifying factors which function together to render it dominant to the mutant. Dominance in this case must have been evolved since the separation of Sea Island from the other New World cottons. 
(b) In my previous paper I mentioned the exceptional ease presented by poultry, in which a large number of the characters which distinguish the domesticated breeds from the wild form are non-lethal dominants. The case was left aside as unexplained, not that the difficulty it offered was opposed especially to my own views, but being on any view exceptional it would necessarily require a special explanation. It was not obvious to me why this species should offer an exception to the rule to which other animals and plants, including some other birds, appeared to conform. I must now withdraw the statement that the theory of the evolution of dominance by selection appears to throw no light upon the case of poultry; for that theory seems to be capable of doing its full duty of explaining both the rule and the exception.

It is now generally admitted that many mutations which in the wild state were kept rare by counter-selection have been in domestication not merely sheltered from competition, but favored by man for their novelty. The mutant forms have attracted man's interest, are valued by him, and form the distinctive characters of many domesticated breeds. In most species the novelties appear as recessive segregates, and can at once be bred true. It appears to me that the exceptional circumstances needed to explain the case of Gallus are to be sought, not in the wild species, but in the conditions of its domestication. For, unlike other domestic animals and plants, the domestic hen is, in its own country, constantly liable to be mated by wild cocks; this is frequent in India to the present day and must have been the prevalent condition especially in the early stages of domestication by jungle tribes. In these circumstances the only mutants which could be established in the domestic flock would be those in which the heterozygote differed from the first somewhat from the wild type; that is, mutations which were not completely recessive. Moreover, these heterozygotes would very frequently appear in domestic broods mixed with chicks of the wild type, and the distinctions of the breed could only be maintained by human selection. Such selection would necessarily favor those which differed most clearly from the wild type, or in fact, those in which the mutation was least recessive, or most dominant.

This case of human selection for dominance of the mutant differs from the natural selection for dominance of the wild type 
in that the whole population exposed to selection consists of heterozygotes, instead of only one in some five or ten thousand. Its evolutionary effect, in the absence of inbreeding, will therefore be correspondingly rapid, and it is not surprising that great changes should be produced in a thousand generations, or even much less, especially in view of the statement (Punnett, "Heredity in Poultry," 91-94) that several of these very mutants, among comb characters, for example, both Pea and Duplex, show very variable degrees of dominance in different breeds.

It will be observed that this explanation covers both the high proportion of non-lethal dominants, and the high degree of dominance shown by them in the breeds of domestic poultry; it is moreover capable of direct experimental verification, for it does not involve the corresponding modification of the wild species. The crucial test would consist in crossing any one of these dominants, such as dominant White, continually back into a line of genuinely wild jungle fowl; the White gene should then, on the view developed above, lose its dominance to an appreciable extent in a few generations, and when the homozygous White was reconstituted in birds principally of wild ancestry, it should be appreciably different from the heterozygotes in the same stock. White would not, however, in my view be expected ever to become a complete recessive in such an experiment.

In several ways the theory of the evolution of dominance throws light upon evolutionary problems. It is evidently quite unnecessary to view the ordinary recessive mutation as evidence of the "dropping out" of the original gene, or to regard the case of poultry as exceptional in that "something new has been added." The assumption, always plausible but not positively helpful to the problem presented by the dominant mutants, that our domestic breeds are descended from more than one species of Gallus, is now evidently superfluous in this connection.

In regard to mutation rates the case of cotton shows at once that with the evolution of new species new mutations may commence to occur, or at least to occur with appreciable frequency. While, on the other hand, the large number of mutations which are almost completely recessive implies, in view of the slowness with which dominance must in most cases be modified, that many unfavorable mutations have persisted in occurring for an immense period. 
Perhaps the most illuminating sidelight of all which it throws upon evolutionary theory arises from the fact that it reveals an effect of natural selection which has nothing to do with the adaptation of the species to the conditions of its environment. The work of adaptation has hitherto seemed to be the only one upon which natural selection is engaged, and nothing could be more difficult to measure than achievement in this respect. It has now been shown that the same agency, as a minute by-product of its activity, must also tend to modify dominance, and, if the recessiveness of each several mutation be referred to this cause, the vast number of reactions which must have been so modified gives a measure of its efficacy, which might have startled even a Weismann.

ROTHAMSTED, Aug. 22, 1928

R. A. FISHER 